IRSTI 29.03.85

\author{
1,2B.A. Iskakov, ${ }^{1,2}$ Y.M. Tautayev, ${ }^{1,2}$ T.X. Sadykov, \\ ${ }^{3,4}$ A.L. Shepetov, ${ }^{3,5}$ N.M. Salikhov \\ ${ }^{1}$ Satbayev University, Institute of Physics and Technology, Almaty, Kazakhstan \\ ${ }^{2}$ Al-Farabi KazNU, Almaty, Kazakhstan. \\ ${ }^{3}$ Tien Shan Scientific Station, Almaty, Kazakhstan \\ ${ }^{4}$ P.N. Lebedev Institute of Physics of RAS, Moscow, Russia \\ ${ }^{5}$ Institute of Ionosphere, Almaty, Kazakhstan
}

\title{
Development and creation of a software system for the monitoring system mac1
}

\begin{abstract}
A special system of acoustic detectors was created to search for possible correlations between wide air showers and the signal of elastic oscillations from the depths of the earth's crust. They are intended for joint synchronous work with storm installation.
\end{abstract}

Key words: microphone, signals, microcircuit, program, acoustics.

A preliminary search for short-term acoustic emission signals in events related to the group passage of high-energy muons was carried out in a special experiment at the Tien-Shan high-altitude station in 2012. Upon completion of the modification of the Tien-Shan stormwater installation and the transition to regular recording of wide atmospheric showers, experiments of this kind it is expected to continue in full.

A highly sensitive microphone with a sensitivity of $20 \mathrm{mV} / \mathrm{Pa}$ in the acoustic frequency range of $500-1000 \mathrm{~Hz}$ is located at a depth of $50 \mathrm{~m}$ from the ground surface inside a well drilled in the rocky soil. The distance between the well and the storm detector system is approximately $200 \mathrm{~m}$. The circuit of electronic equipment which provides registration is shown in Figure 1. The transmission of electrical signals from a microphone from the depth of a well is made over a cable line formed by a twisted pair of wires through a transformer junction. The microphone and the signal-carrying intermediate small-sized transformer are a single building block, which is completely lowered into the well. A constant voltage of $\pm 3 \mathrm{~V}$ for powering the microphone is produced by an independent power source, which is built on the basis of a separate transformer with an ungrounded secondary winding and does not have direct electrical contact with the rest of the electronic circuit or the power lines of the external electrical network. From the power source to the microphone, this voltage is applied through the second pair of twisted wires. Thus, the microphone node of the measuring system is electrically isolated from all common ground lines and power supply to the electronic circuits.

Acoustic detector signals are recorded in a special room, which is located directly at the upper edge of the well. In the room are the other nodes forming the signal equipment. The differential amplifier (element D1 in Figure 1) provides 100 times the amplification of the useful signal while simultaneously suppressing common mode noise that occurs on a long link. The common mode rejection ratio of this type of amplifier is $\sim 70 \mathrm{~dB}$. At the output of the differential amplifier, bipolar sinusoidal signals are formed, belonging to the acoustic range $(\sim 102-104 \mathrm{~Hz})$ and ready for digitization by means of an ADC system. The lowfrequency selector built on the operational amplifiers D2 and D3 serves to highlight the modulating amplitude of the acoustic signal of the low-frequency envelope, which is provided for registration through a separate ADC channel. 
A special small-scale low-power ADC system has been developed for recording acoustic detector signals. It is placed together with the formation schemes directly at the upper edge of the well. The ADC system is based on the Raspberry $\mathrm{PiB}+$ single-board computer on a Broadcom BCM2835 type microprocessor with a clock frequency of 700 $\mathrm{MHz}$. This computer, through the lines of a generalpurpose digital I/O port embedded in it, controls two ADC elements with AD7887 chips manufactured by
Analog Devices. The clock sequences C (clock) and $\mathrm{CS}$ required for the operation of the microcircuits are generated by a computer microprocessor running a special driver program. The same program accepts the results of converting the input signals that come from the DO outputs of the ADC chips in the form of a serial binary code and converts them into binary data bytes. A schematic diagram of the connection of the $\mathrm{ADC}$ elements to the Raspberry $\mathrm{PiB}+$ microcomputer is shown in Figure 2.

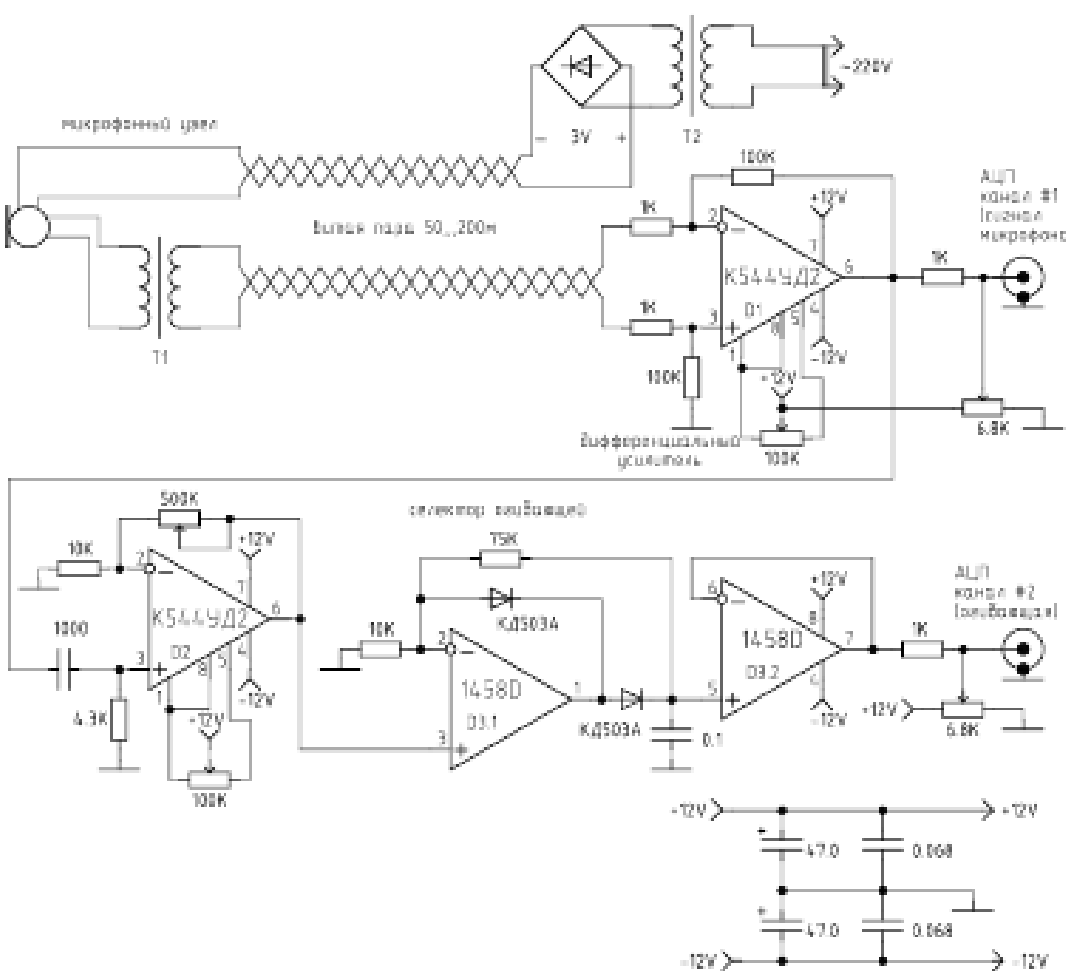

Figure 1 - Connection diagram of a microphone in an acoustic detector

The signals of the ADC microcircuits are connected to the pins of the GPIO port of the microcomputer through the buffer elements of the TTL logic. They are part of the K155LN1 chip and through resistive dividers, which are necessary for matching the level of logic signals from the microprocessor with the level of the AD7887 chip. The input analog signals are recorded over two information channels of the small-sized ADC system continuously with a period of $2 \mathrm{~ms}$. Measured data is collected for subsequent on-line analysis in a file on a local disk that connects to the Raspberry $\mathrm{PiB}+$ microcomputer via its built-in USB interface.
The block diagram of the software package that is used to record the data of the acoustic detector is shown in Figure 3. As can be seen in this diagram, the program interacts directly with the hardware registers built into the Raspberry PiB microcomputer parallel I/O port, to the lines of which the control signals of the ADC chip are connected k09raspi.c driver. This program is written in the compiled programming language $\mathrm{C}$ and is responsible for the formation, in accordance with the corresponding temporal characteristics, of two clock sequences C and CS. They provide the necessary synchronization of the ADC chips. In response to each clock cycle of 
the synchronization sequence $\mathrm{C}$, the next bits of the data code are received from the microcircuits, which are registered by the k09raspi.c program. on the two input lines of the parallel port. The received data bits are processed by the driver software, and parallel bytes of the 12-bit ADC code are formed from them, which are placed by this driver into a segment of shared memory that is readable by other programs.

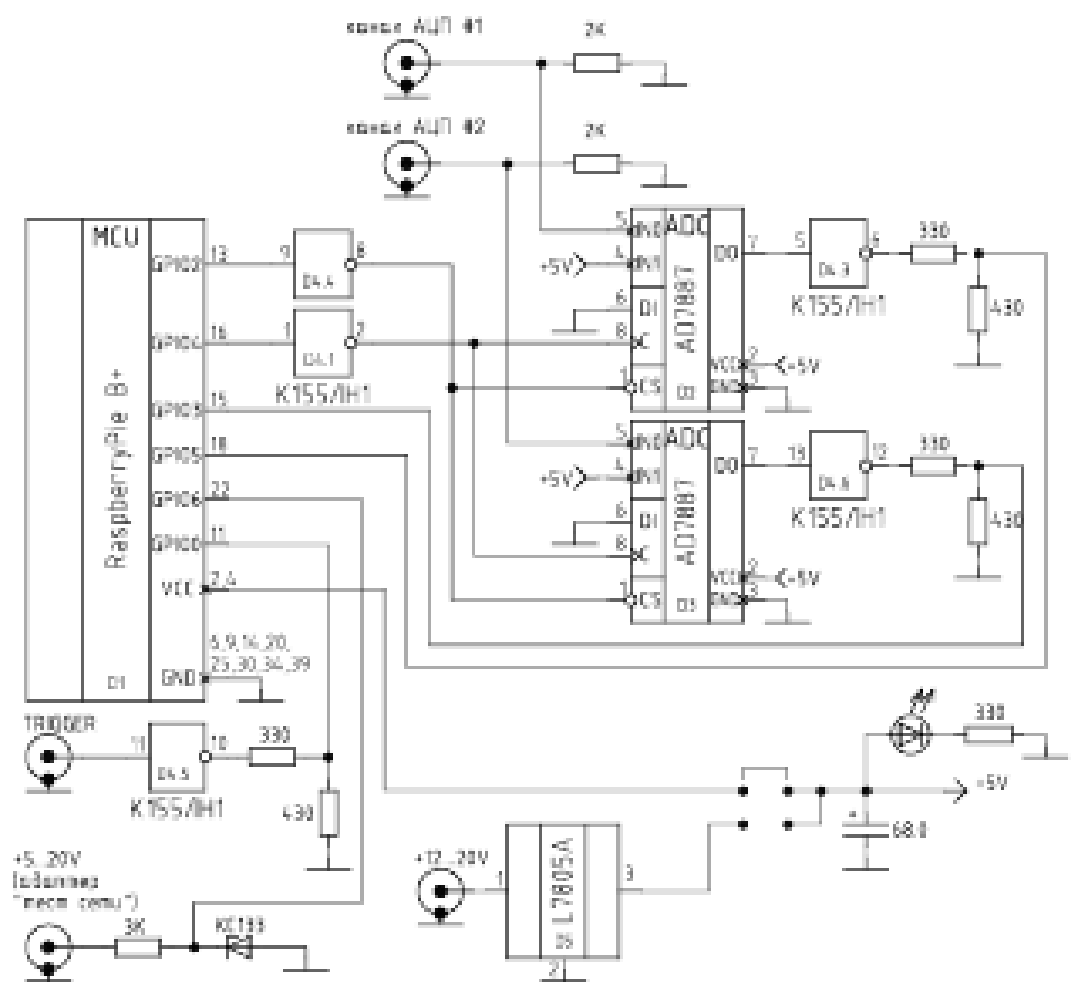

Figure 2 - Compact ADC system

Responsible for sampling the data generated by the driver from shared memory and further processing this information is the measurement management program k09001. This program performs the standard functions of supporting the measurement process, reads, immediately after launch, a set of configuration parameters from the input file k09in. It adjusts the algorithm of its work to the specifics of a specific measurement setup, tracks the appearance of new information packages in the shared memory segment, generates an array of binary data with measurement results from these packages, and writes this array to a file with the .zdat extension on the local disk. Before writing to the disk, the array is compressed by the archiver integrated into the program $\mathrm{k} 09001$, which reduces the total amount of output data and shortens the information exchange with the disk. Since the files recorded in this way directly during the measurements are packed in an irregular way, before they are sent for permanent storage they are processed once again by a special program - the utility k09006. It converts them into regular archive files .dat.gz, which can be read by standard archivers such as zip, gzip and the like. After the conversion, the data archive files are automatically sent to the general processing center via wireless communication lines.

Measurement management program k09001 is written in $\mathrm{C}++$ compiled programming language, which allows to satisfy rather stringent requirements for its speed. In addition, the required processing speed of incoming information is achieved due to double buffering and parallelization of operations in this program. When it starts, it creates two buffer arrays in the computer's memory to write data, at each moment of time one of these arrays is filled with current data. Information from the second at the same time is being written to the computer disk in 
a separate stream of execution. The Raspberry PiB

+ microcomputer, which provides the process of digitizing the signals of an acoustic detector, runs on a specially adapted version of the Linux operating system. The programs included in this system ensure the performance of normal operations to support the normal operation of the entire measurement process: automatic start of the necessary utility programs, monitoring and automatic correction of the system clock, the ability to remotely connect via communication lines to monitor the system operation on-line and etc.

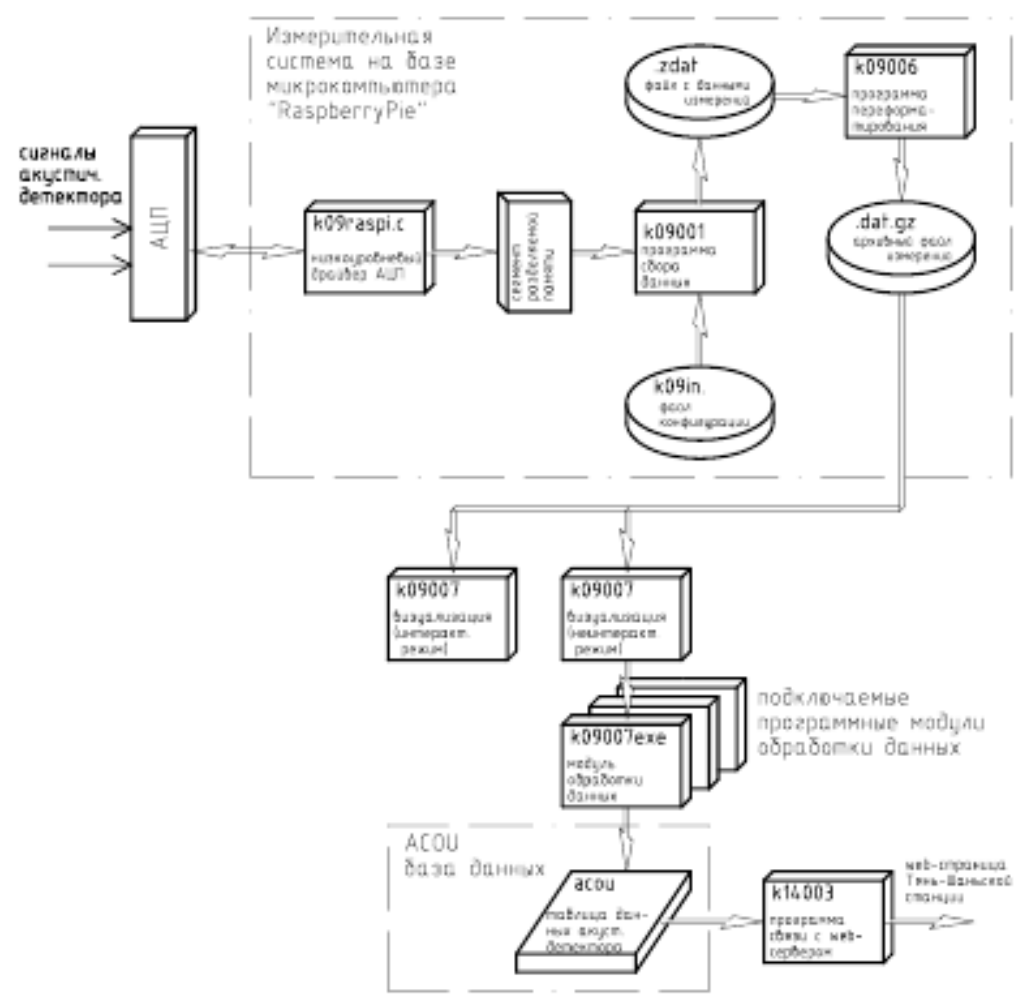

Figure 3 - Block diagram of the software system for the monitoring system

One of the specialized programs designed to work with the archived data files of the acoustic detector is the visualization program $\mathrm{k} 09007$, which, when launched in graphical mode of operation, allows you to view the measurement results stored in these files in the form of interactive graphs. The program k09007 is written in Python, and its graphical window interface is implemented using the Tkinter library, which is included in the standard distribution of this language. Graphs are drawn in the program window by means of the Python graphical library Matplotlib.

In addition to the interactive user mode of direct interaction with the user, the k09007 program also supports a non-interactive mode, in which information from archive files with measurement results, instead of directly displayed in the graphics window, is transmitted to external plug-in modules k09007exe, which implement various data processing algorithms, search for short peaks of intensity, etc. In particular, one of these modules is used to calculate the averaged parameters of the recorded signals: the rms values of the ADC codes, which are calculated both for the analog signal removed from the microphone and for its low-frequency envelope, as well as the number of events recorded per minute with ADC codes exceeding a number of threshold values.

\section{Conclusion}

As shown in the block diagram (Figure 3), the averaged parameters of the acoustic signal calculated in this way are loaded by the program k09007 into the special table for the acou database of the same name. The information stored in this table can be requested 
by the web server to be displayed in text or graphic form on the Tian-Shan station web page. Processing requests from the web server and preparing for it the necessary information is made through an auxiliary program "CGE-general-purpose script k14003.

\section{References}

1. Berger Ch. et al. (Frejus Collab.). "Experimental study of muon bundles observed in the Fréjus detector. "Physical Review D, 40, 7 (1989): 2163.

2. Beringer, J., Arguin, J. F., Barnett, R. M., Copic, K., Dahl, O., Groom, D. E., ... \& Yao, W. M. "Review of Particle Physics. " Physical Review D 86 (2012): 1-22.

3. Young, J.B., Presgrave, B.W., Aichele, H., Wiens, D.A. and Flinn, E.A. "The Flinn-Engdahl Regionalisation Scheme: the 1995 revision. "Physics of the Earth and Planetary Interiors 96, 4 (1996): 223-297.

4. Shepetov, A. L., Sadykov, T. K., Mukashev, K. M., Zhukov, V. V., Vil'danova, L., Salikhov, N. M., ... \& Argynova, A. K. "Seismic signal Registration with an acoustic detector at the Tian Shan Mountain Station. "News of the National Academy of Sciences of the Republic of Kazakhstan - Series of geology and technical sciences 3, 429 (2018): 47-56.

5. Ambrosio M. et al. (MACRO Collab.). "Vertical muon intensity measured with macro at the Ggran Sasso laboratory. " Physical Review D 52, 7 (1995): 3793-3802.

6. Gusev G. A., Zhukov V. V., Merzon G. I. et al. "Cosmic rays as a new instrument of seismological studies.” Bull. Lebedev Phys. Inst. 38, 12 (2011): 374-379.

7. Tribedy P. Journal of Physics: Conference Series Ser. 832 (2017) 012013.

8. Kroemer O. 2009 Proceedings of the 31 st ICRC.- p. 25

9. Adamson P. et al. 2007 Phys. Rev. -Vol. D76. -P. 052003.

10. Beisembayev U., Dalkarov O.D., Sadykov T.Kh. et.al. Nuclear Instruments and Methods in Physics Research A. V.832 2016-p.158-178.

11. G. V. Kulikov and G. B. Khristiansen. 1959 Soviet Physics JETP 35(8):441-444.

12. J. Abraham, Pierre Auger Collaboration 2010 Physics Letters B - V685. - p. 239-246.

13. M. Witek 2017 EPJ Web of Conferences 141, 01007. 